\title{
THE ICGA ACTIVITIES IN TURIN, ITALY
}

\author{
P Clancarın ${ }^{1}$ \\ Turın, Italy
}

The $14^{\text {th }}$ World Computer-Chess Championship and the Computer Olympiad will take place from May 24 Junı 1, 2006 at the Oval and the CG 2006 on May 29-31, 2006 at the Aula Magna of the University of Turın The local organising committee consists of Paolo Ciancarını, Maurızıo Molınaro, and Edoardo Calıa We are supported by our secretarial staff and by the ICGA Headquarters in Maastricht, The Netherlands Moreover, we are in close contact with ICGA President David Levy (WCCC and CO) and with Yngvi Bjornsson

\begin{tabular}{|c|c|c|c|c|}
\hline Date & & $\begin{array}{l}\text { WCCC } \\
\text { At the Oval }\end{array}$ & $\begin{array}{l}\text { CG2006 } \\
\text { At the University }\end{array}$ & $\begin{array}{l}\text { Olympiad } \\
\text { At the Oval }\end{array}$ \\
\hline $\begin{array}{l}\text { May 24, } \\
\text { Wednesday }\end{array}$ & & Arrıval & & Arrıval \\
\hline 25, Thursday & $\begin{array}{l}13-18 \mathrm{~h} \\
19-24 \mathrm{~h}\end{array}$ & $\begin{array}{l}\text { Testıng, Openıng and Players } \\
\text { meetıng } \\
\text { Round } 1\end{array}$ & & $13-24 \mathrm{~h}$ \\
\hline 26, Friday & $\begin{array}{l}13-18 \mathrm{~h} \\
19-24 \mathrm{~h}\end{array}$ & $\begin{array}{l}\text { Round } 2 \\
\text { Round } 3\end{array}$ & & $13-24 \mathrm{~h}$ \\
\hline 27, Saturday & $\begin{array}{l}13-18 \mathrm{~h} \\
19-24 \mathrm{~h}\end{array}$ & $\begin{array}{l}\text { Round } 4 \\
\text { Round } 5\end{array}$ & & $13-24 \mathrm{~h}$ \\
\hline 28 , Sunday & & Excursion day & & Excursion day \\
\hline 29, Monday & $\begin{array}{l}13-18 \mathrm{~h} \\
19-24 \mathrm{~h}\end{array}$ & $\begin{array}{l}\text { Round } 6 \\
\text { Speed tournament }\end{array}$ & $830-1230$ & $13-24 \mathrm{~h}$ \\
\hline 30, Tuesday & $\begin{array}{l}13-18 \mathrm{~h} \\
19-24 \mathrm{~h}\end{array}$ & $\begin{array}{l}\text { Round } 7 \\
\text { Round } 8\end{array}$ & $830-1230$ & $13-24 \mathrm{~h}$ \\
\hline 31, Wednesday & $\begin{array}{l}13-18 \mathrm{~h} \\
19-24 \mathrm{~h}\end{array}$ & $\begin{array}{l}\text { Round } 9 \\
\text { Round } 10\end{array}$ & $830-1230$ & Play-offs \\
\hline $\begin{array}{l}\text { June 1, } \\
\text { Thursday }\end{array}$ & $\begin{array}{l}13-18 \mathrm{~h} \\
19-24 \mathrm{~h}\end{array}$ & $\begin{array}{l}\text { Round } 11 \\
\text { Play-off and Closing dınner }\end{array}$ & Closing dinner & Closing dinner \\
\hline
\end{tabular}

More information will be published in the March 2006 issue See also our website www icga org

\section{THE ICGA JOURNAL REFEREES OF 2005}

\section{The Editorlal Board}

The Editor-1n-Chief and the Editorial Board would like to acknowledge the expert assistance of the following persons in refereeing submissions to the Journal during the year 2005 We hope to meet an equal willingness among the referees for the years to come

$\begin{array}{lll}\text { Yngo Althofer } & \text { Jeroen Donkers } & \text { Hans Kuiff } \\ \text { Yngvi Bjornsson } & \text { Markus Enzenberger } & \text { David Levy } \\ \text { Ur1 Blass } & \text { Haw-ren Fang } & \text { Ulf Lorenz } \\ \text { Bruno Bouzy } & \text { David Fotland } & \text { Shaul Markovitch } \\ \text { Dennis Breuker } & \text { Reijer Grimbergen } & \text { Frans Morsch } \\ \text { Marc Brockıngton } & \text { Dap Hartmann } & \text { Dana Nau } \\ \text { Arıe de Bruin } & \text { Ryan Hayward } & \text { Gian-Carlo Pascutto } \\ \text { Shay Bushınsky } & \text { Guy Haworth } & \text { Jacques Pitrat } \\ \text { Tristan Cazenave } & \text { Bernard Helmstetter } & \text { Christian Posthoff } \\ \text { Guillaume Chaslot } & \text { Ka1 Himstedt } & \text { Jan Romein } \\ \text { Ken Chen } & \text { Bob Hyatt } & \text { Jack van Rijswijck } \\ \text { Remı Coulom } & \text { Akıhıro Kishımoto } & \text { Jahn Saito } \\ \end{array}$

\author{
Jonathan Schaeffer \\ Nathan Sturtevant \\ Omıd David Tabibi \\ Pascal Tang \\ Ed Trice \\ Erık van der Werf \\ Mark Winands \\ Bob Wirth \\ Eng Kion Wong \\ Wu Jing Yang
}

\footnotetext{
${ }^{1}$ Dipartımento dı Scienze dell'Informazıone, Unıv di Bologna, Mura Anteo Zambonı 7, 47127 Bologna, Italy Emaıl cranca@cs unibo it
} 